Subscriber access provided by QUEENS UNIV BELFAST

\title{
Article
}

\section{Graphene as Barrier to Prevent Volume Increment of Air Bubbles Over Silicone Polymer in Aqueous Environment.}

Ruben Bartali, Andrea Lamberti, Stefano Bianco, Candido Fabrizio Pirri, Manoj Tripathi, Gloria Gottardi, Giorgio Speranza, Erica lacob, Nicola M. Pugno, and Nadhira Laidani

Langmuir, Just Accepted Manuscript • DOI: 10.1021/acs.langmuir.7b02915 • Publication Date (Web): 18 Oct 2017

Downloaded from http://pubs.acs.org on October 24, 2017

\section{Just Accepted}

"Just Accepted" manuscripts have been peer-reviewed and accepted for publication. They are posted online prior to technical editing, formatting for publication and author proofing. The American Chemical Society provides "Just Accepted" as a free service to the research community to expedite the dissemination of scientific material as soon as possible after acceptance. "Just Accepted" manuscripts appear in full in PDF format accompanied by an HTML abstract. "Just Accepted" manuscripts have been fully peer reviewed, but should not be considered the official version of record. They are accessible to all readers and citable by the Digital Object Identifier (DOI@). "Just Accepted" is an optional service offered to authors. Therefore, the "Just Accepted" Web site may not include all articles that will be published in the journal. After a manuscript is technically edited and formatted, it will be removed from the "Just Accepted" Web site and published as an ASAP article. Note that technical editing may introduce minor changes to the manuscript text and/or graphics which could affect content, and all legal disclaimers and ethical guidelines that apply to the journal pertain. ACS cannot be held responsible for errors or consequences arising from the use of information contained in these "Just Accepted" manuscripts. 


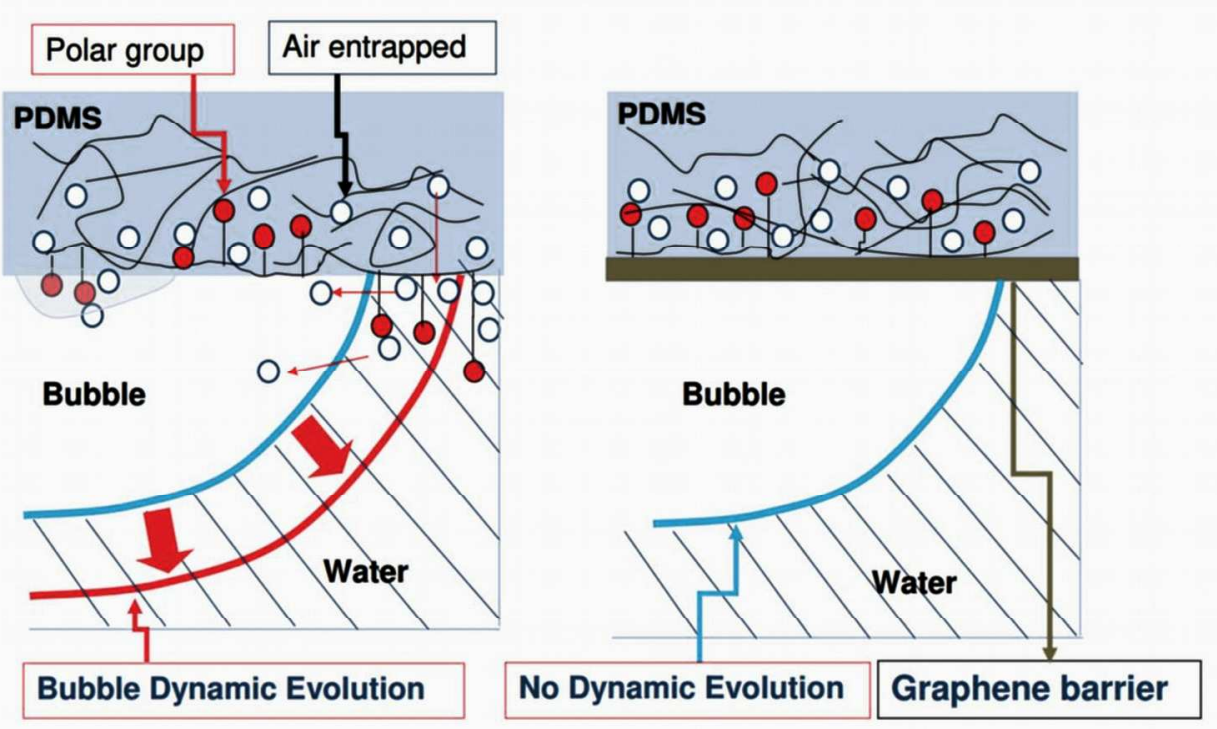

graphical abstract

$159 \times 90 \mathrm{~mm}(300 \times 300 \mathrm{DPI})$ 


\section{Graphene as Barrier to Prevent Volume Increment of Air Bubbles Over Silicone Polymer in Aqueous Environment.}

Ruben Bartali ${ }^{*}, \dagger$, , Andrea Lamberti ${ }^{\S}$, Stefano Bianco ${ }^{\S}$, Candido F. Pirri ${ }^{\S}$, Manoj Tripathi ", Gloria Gottardi $^{\dagger}$, Giorgio Speranza ${ }^{\dagger}$, Erica Iacob ${ }^{\dagger}$, Nicola Pugno ${ }^{\|, \perp, \infty}$, and Nadhira Laidani ${ }^{\dagger}$

${ }^{\dagger}$ Fondazione Bruno Kessler,Center for Materials and Microsystems, via Sommarive 18, 38123 Povo, Trento, Italy

${ }^{\ddagger}$ Dipartimento di Fisica , Università di Trento, via Sommarive 14, 38123 Povo, Trento, Italy

${ }^{\S}$ Applied Science and Technology Department, Politecnico di Torino, Corso Duca degli Abruzzi 24, Turin, IT-10129, Italy

"Laboratory of Bio-Inspired and Graphene Nanomechanics, Department of Civil, Environmental and Mechanical Engineering, University of Trento, 38123, Trento - Italy

${ }^{\perp}$ School of Engineering and Materials Science, Queen Mary University of London, Mile End Road, E1 4NS London - United Kingdom

${ }^{\infty}$ Ket Lab, Edoardo Amaldi Foudation, Italian Space Agency, Via del Politecnico snc, 00133 Rome, Italy

Corresponding author: Ruben Bartali, bartali@fbk.eu, via Sommarive 18 38123, Trento (Italy) 


\begin{abstract}
The interaction of air bubbles with surfaces immersed in water is of fundamental importance in many fields of application ranging from energy to biology. However, many aspects of this topic such as the stability of surfaces in contact with bubbles remain unexplored. For this reason, in this work, we investigate the interaction of air bubbles with different kinds of dispersive surfaces immersed in water. The surfaces studied were polydimethylsiloxane (PDMS), graphite and single layer graphene/PDMS composite. X-ray photoelectron spectroscopy (XPS) analysis allows determining the elemental surface composition while Raman spectroscopy was used to assess the effectiveness of graphene monolayer transfer on PDMS. Atomic force microscopy (AFM) was used to study the surface modification of samples immersed in water. The surface wettability has been investigated by contact angle measurements, and the stability of the gas bubbles was determined by captive contact angle (CCA) measurements. CCA measurements show that the air bubble on graphite surface exhibits a stable behaviour while, surprisingly, the volume of the air bubble on PDMS increase as function of immersion time (bubble dynamic evolution). Indeed, the air bubble volume on the PDMS rises by increasing immersion time in water. The experimental results indicate that the dynamic evolution of air bubble in contact with PDMS is related to the rearrangement of surface polymer chains via the migration of the polar groups. On the contrary, when graphene monolayer is present on PDMS it acts as absolute barrier suppressing the dynamic evolution of the bubble and preserving the optical transparency of PDMS.
\end{abstract}

Corresponding author: Ruben Bartali, bartali@fbk.eu, via Sommarive 18 38123, Trento (Italy)

Keywords: protective layer, PDMS, captive contact angle, wettability, graphite, 2D material 


\section{Introduction}

Wettability of surfaces is widely studied in literature because the wetting characteristic of materials is crucial for many applications such as microfluidics, self-cleaning surfaces, water-repellent surfaces, bacterial adhesion, ophthalmic membranes, and microelectronic ${ }^{1-11}$. Moreover, liquid-solid interaction has attracted scientific interest in the field of nanoscience e,g Rafiee et al. studied the water transparency of 2D materials ${ }^{12,13}$. Most scientific studies focused on the interaction of water drops on a surface and in air environment; only a few papers studied the complementary system composed by a bubble of gas in contact with surfaces immersed in liquid media. ${ }^{14,15}$. However, bubble in contact with surface immersed in liquid could have an enormous impact in many fields, for example, on the embolism evolution investigation. Avoiding the intravascular gas bubble is vital to the health. Indeed, embolism is one of the main causes of neurocognitive dysfunction in cardiopulmonary surgery. Suzuki and Eckmann studied the adhesion force of air bubbles in the microvessel (arteriole) of mesenteric tissue ${ }^{16}$. They observed that the adhesion force depends residence time and on the presence of endothelium, thus being related to the physic-chemical structure of the internal surface. Another interesting example where the bubbles in contact with a surface can play an important role is a direct methanol fuel cell. Indeed, the behaviour of the $\mathrm{CO}_{2}$ bubbles in contact with materials immersed in liquids is a key factor to improve the performance of the device ${ }^{17}$. The high methanol flow rate results in small discrete $\mathrm{CO}_{2}$ gas bubbles and short gas slugs that increase the cell performance. However, the adhesion of the gas bubbles on the surface of materials immersed in water is not entirely understood, and an example is the unexpected nanobubbles stability on graphite immersed in water ${ }^{18,19}$. The thermodynamic properties, stability, the role of surface structure/chemistry and the role of chemical nature of the bubbles in many liquid-solid systems are still poorly understood.

The intermolecular forces in the gasses are due to the non-bonding interactions as induced dipoleinduced dipole interactions, known as dispersion force ${ }^{20}$. In order to study an homogeneous system, herein we exploit captive bubble contact angle measurements (see supplementary information) to characterize the interaction of the gas bubbles with different kinds of dispersive surfaces: PDMS (polymer chains), graphite (rigid honeycomb structure), and graphene on PDMS (honeycomb structure + polymer chains). PDMS and graphite are reported in the literature as dispersive surfaces, and they are interesting from the technological point of view because of being widely used in many applications and studies ${ }^{21-24}$. The PDMS, in particular, is used as an electrical isolator, as a structural material for microfluidic devices, or as a gas-permeable membrane ${ }^{25,26}$. In many applications, PDMS remains for a long time immersed in contact with water and often in contact 


\section{Experimental Section}

\section{Materials}

PDMS membranes were prepared by mixing the polymer base and the curing agent (Sylgard 184, Dow Corning) with 10:1 mixing ratio (oligomer: curing agent) and degassing in low vacuum for $1 \mathrm{~h}$. The mixture was then poured into PMMA molds with an area of $2 \mathrm{~cm}^{2}$ and depth of $1 \mathrm{~mm}$ (fabricated by milling machine) and cured in a convection oven following two different thermal treatment. One set of samples was cured for 1 hour at $60^{\circ} \mathrm{C}$ (bare PDMS sample) while another set was cured for $30 \mathrm{~min}$ at $60 \mathrm{C}$. This soft curing step was performed to allow a partial crosslinking of the material, that produces a soft hardening of the membrane. As already reported by Lamberti et $a l .{ }^{26}$ This strategy allows facilitating the subsequent bonding of the PDMS membrane with other surfaces, permitting the later graphene transfer. The growth of single-layer graphene is performed using a cold-wall Chemical Vapor Deposition (CVD) system. The synthesis procedure is carried out on high-quality copper foils and foresees the catalytic decomposition of carbon precursor $\left(\mathrm{CH}_{4}\right)$ in a high-temperature deposition process $\left(1000{ }^{\circ} \mathrm{C}\right)$ in the reactive $\mathrm{H}_{2} / \mathrm{Ar}$ atmosphere $\left(\mathrm{Ar} / \mathrm{H}_{2} / \mathrm{CH}_{4}\right.$ partial pressure: 80:10:10). The growth process can guarantee the controlled formation of single-layer graphene with reduced defectiveness. The graphene/Cu substrate is transferred on the partially reticulated PDMS surface, obtaining an excellent adhesion between the elastomeric substrate and 
the $\mathrm{G} / \mathrm{Cu}$ film during the final reticulation procedure performed for other $30 \mathrm{~min}$ at $60^{\circ} \mathrm{C}$. Cu layer is then removed in acidic $\mathrm{FeCl}_{3}$ solution in water (2.25 $\mathrm{M}$ for $1 \mathrm{~h}$ ).

Graphite samples were prepared by mechanical exfoliation of Highly Oriented Pyrolytic Graphite (HOPG) graphite (Goodfellow Cambridge). The thickness of exfoliated graphite was 14 microns; the lateral size was $10 \mathrm{~mm} \mathrm{X} 10 \mathrm{~mm}$, and the resistivity was $8 * 10^{-5} \mathrm{Ohm} * \mathrm{~cm}$. The contact angle on the PDMS and graphene/PDMS surface has been measured on the sample as received (aged surfaces).

\section{Methods}

The surface free energy was calculated by contact angle measurements following Owens-Wendt approach ${ }^{54}$. The total surface energy $\gamma \mathrm{s}$ in the Owens-Wendt approach is defined as the sum of polar and dispersive components indicated as $\gamma_{s}^{p}$ and $\gamma_{s}{ }^{d}$, respectively:

$\gamma_{s}=\gamma_{s}^{p}+\gamma_{s}^{d}$

where, the dispersive component is due to London interactions and polar components are the sum of hydrogen, polar, inductive and acid-base interactions. By the Owens-Wendt method, the surface energy can be estimated by the following equation:

$$
(1+\cos \theta) \gamma_{l}=2 \sqrt{\gamma_{s}^{d} \gamma_{l}^{d}}+2 \sqrt{\gamma_{s}^{p} \gamma_{l}^{p}}
$$

Where $\theta$ is the contact angle, $\gamma_{l}$ the total surface tension of liquids, $\gamma_{l}^{d}$ is the dispersive component of the fluids and $\gamma_{l}^{p}$ is a polar component of the fluids. Due to two unknown parameters $\left(\gamma_{s}^{p}\right.$ and $\left.\gamma_{s}^{d}\right)$ in Equation 1, the contact angle has to be measured using two liquids with known properties, one dispersive liquid and one polar liquid. In this study, static contact angle measurements for surface energy estimation were performed with milliQ deionized water and diiodomethane. These two liquids have been used since diiodomethane has only dispersive component, water has a dominant polar component. The total surface energy of liquids and their polar and non-polar components are listed in Table 1.

Table 1. Surface energy components of the probe liquids $\left(\mathrm{mJ} / \mathrm{m}^{2}\right) .{ }^{55}$

\begin{tabular}{lccc}
\hline Liquids & \multicolumn{1}{c}{$\gamma_{1}$} & \multicolumn{1}{c}{$\gamma_{1}{ }^{\mathbf{d}}$} & $\gamma_{1}^{\mathbf{p}}$ \\
\hline Water & 72.8 & 21.8 & 51 \\
Diiodomethane & 52.8 & 52.8 & $\approx 0$ \\
Total surface tension $\gamma_{1}$, the dispersive components $\boldsymbol{\gamma}_{1}^{\mathbf{d}}$ and the \\
polar components $\boldsymbol{\gamma}_{1}^{\mathbf{p}}$ of the liquids in $\mathbf{m J} / \mathbf{m}^{2}$. \\
\hline
\end{tabular}




\section{Result and Discussion}

The surface chemistry of exfoliated graphite (EG) and polydimethylsiloxane (PDMS ) provided by XPS analysis is summarized in Table 2. The survey spectra of XPS on EG show, as expected, the presence of carbon and a small amount of oxygen, due to contamination. The surface chemistry of the PDMS indicates the presence of Carbon, Oxygen and Silicon. The percentage of carbon and oxygen on PDMS is less than the theoretical value (50 and $25 \%$, respectively) while the proportion of silicon is slightly higher of theoretical one (i.e. 15\%). The unbalanced surface stoichiometry is typical of slightly aged PDMS as reported in the literature ${ }^{40}$.

Table 2. Chemical surface composition and surface energy of graphite, PDMS and graphene-PDMS.

\begin{tabular}{lllllll}
\hline & \multicolumn{2}{l}{ XPS } & & \multicolumn{2}{l}{ Surface Energy (Owens-Wendt) $\mathbf{m J} / \mathbf{m}^{2}$} \\
& \multicolumn{2}{l}{ Atomic percentage $\%$} & & & \\
\hline & Carbon & Oxygen & Silicon & Total & Dispersive & polar \\
PDMS & $53.5 \pm 0.5$ & $26.86 \pm 0.3$ & $19.6 \pm 0.2$ & $25.8( \pm 2.5)$ & $25.7( \pm 2.4)$ & $0.1( \pm 0.1)$ \\
Graphite & $98.5 \pm 0.9$ & $1.5 \pm 0.1$ & - & $52.2( \pm 3)$ & $50.8( \pm 2.8)$ & $2.2( \pm 0.2)$ \\
PDMS+Graphene & $54.2 \pm 0.4$ & 26.30 .2 & $19.4 \pm 0.2$ & $33( \pm 2)$ & $32.9( \pm 1.9)$ & $0.1( \pm 0.1)$ \\
\hline
\end{tabular}


Through drop shape angle measurement, we found an average water contact angle of $80^{\circ} \pm 2$ on graphite, typical of slightly aged graphite ${ }^{41}$ and and $107^{\circ} \pm 2$ on PDMS. (the authors underline that some samples of PDMS show a C.A of $105 \pm 2$ ). The diiodomethane, which is a dispersive liquid, showed contact angles lower than $10^{\circ}$ on graphite and higher than $60^{\circ}$ on PDMS. The total surface energy of graphite was $52.2 \mathrm{~mJ} / \mathrm{m}^{2}$, and more of the $95 \%$ of surface energy was due to dispersive components. Only a small component of the surface (4.4\%) derived from the polar component; the polar component was probably due to the contaminations, as corroborated by XPS findings. The PDMS shows a surface energy value lower than graphite, as indicate by the contact angle of diiodomethane, i.e. $25.5 \mathrm{~mJ} / \mathrm{m}^{2}$. Similarly, the graphite has dispersive components on PDMS is more than $99 \%$ of surface energy, and only a negligible percentage of the surface energy is due to polar interaction. The interaction of air bubble with the surface has been studied using captive bubble contact angle. Subsequently, exfoliated graphite was then immersed in water and the interaction of air bubbles with the surface has been studied using CCA. The air captive contact angle on graphite was around $82^{\circ}$ and as already reported by the authors it remains stable as a function of immersion time ${ }^{42}$. The bubble contact angle stability appears in contrast to the trend reported in the literature with sessile contact angles ${ }^{41,43-45}$, since the immersion procedure tends to keep the surface cleaner thus reducing airborne contamination to that deriving from the small volume of the bubble. In the same way, we also estimated the volume of the bubble, using Drop Analysis software ${ }^{53}$.
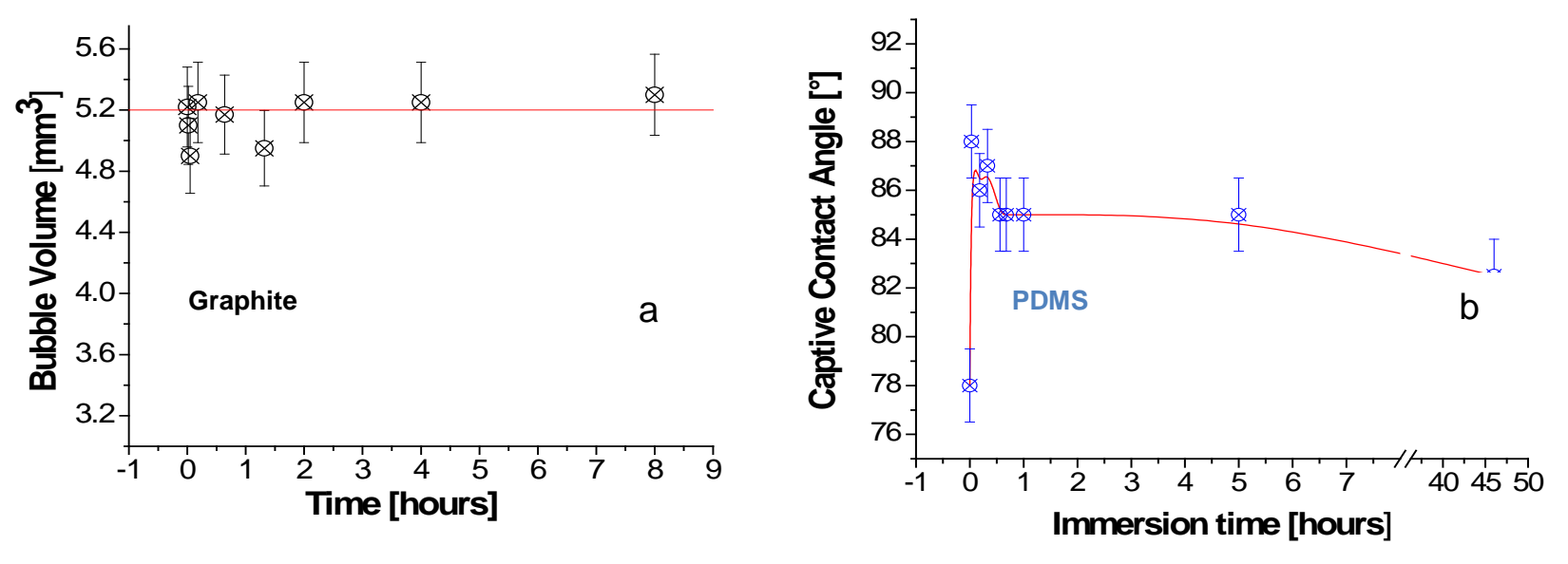

Figure 1. Air bubble volume on (a) graphite and (b) Captive Contact Angle on PDMS as a function of the time.

Figure 1a shows the time evolution of volume of the bubble in contact with graphite surface; the air bubble volume on graphite remains within the experimental error constant. The bubble test has been 
performed on PDMS surface. We observed an increase of the captive contact angle from $78^{\circ}$ to $88^{\circ}$, an instability of contact angle in the first 20 minutes, after an increase and finally a stabilisation of the CCA at $83-84^{\circ}$, see figure $1 \mathrm{~b}$. The initial increase of CCA is probably due to presence of surface contaminations. The reduction in CCA, after initial stage, was expected since Hillborg et al. ${ }^{46,27}$. and Gustavsson et al. ${ }^{28}$ reported the variation of sessile contact angle after the immersion of PDMS in water. They reported that reduction of hydrophobicity on PDMS is due to the migration of the polar group at the surface of polymer when the polymers are immersed in water, moreover the permeation of water induces also the formation of polar Si-OH groups due to hydrolysis of PDMS backbone ${ }^{47}$. In this experiment, surprisingly, we also observed an increase in the bubble volume (see Figure 2a). The starting volume was $1.69 \mathrm{~mm}^{3}$ while after 5 hours the volume became 2.03 $\mathrm{mm}^{3}$, i.e. an increase of $21 \%$. To corroborate this result, the immersion time was extended up to 46 hours. After 46 hours, we measured a contact angle of $82.5^{\circ}$ and a volume of $4.03 \mathrm{~mm}^{3 .}$ that means an increase of $238 \%$. The inset of the figure 2a shows the images of the bubble at the beginning of the test and after 46 hours. The red line in the picture reported in figure 2.a indicates the original bubble profile. The variation of the bubble volume means that there is a source of gas. The source of gas can be endogenous as gas in water or PDMS or exogenous as the air flow from outside of captive bubble cell. Therefore in first approximation the source can be (a) due to the coalescence of microbubbles/ nanobubbles dissolved in water, or (b) it related to surface phenomena of PDMS and/or (c) related to the permeation of the gas from the external side of PDMS exposed to the atmosphere to the side exposed to the water. Considering the experimental conditions, we could exclude the permeation of the gas due to a differential pressure between the external pressure and bubble pressure since inside the bubble there is a small positive overpressure. The overpressure inside the bubble was estimated by Young-Laplace equation and was around 40 mbar $^{48}$. We cannot ignore the fact that the increase of bubble volume can be due to the coalescence of microbubbles but the stability of bubble volume on graphite indicate that the coalescence of micro-nanobubbles in first approximation is negligible. The variation of PDMS captive contact angle, reported in figure 1.b, indicates a change in surface chemistry. 


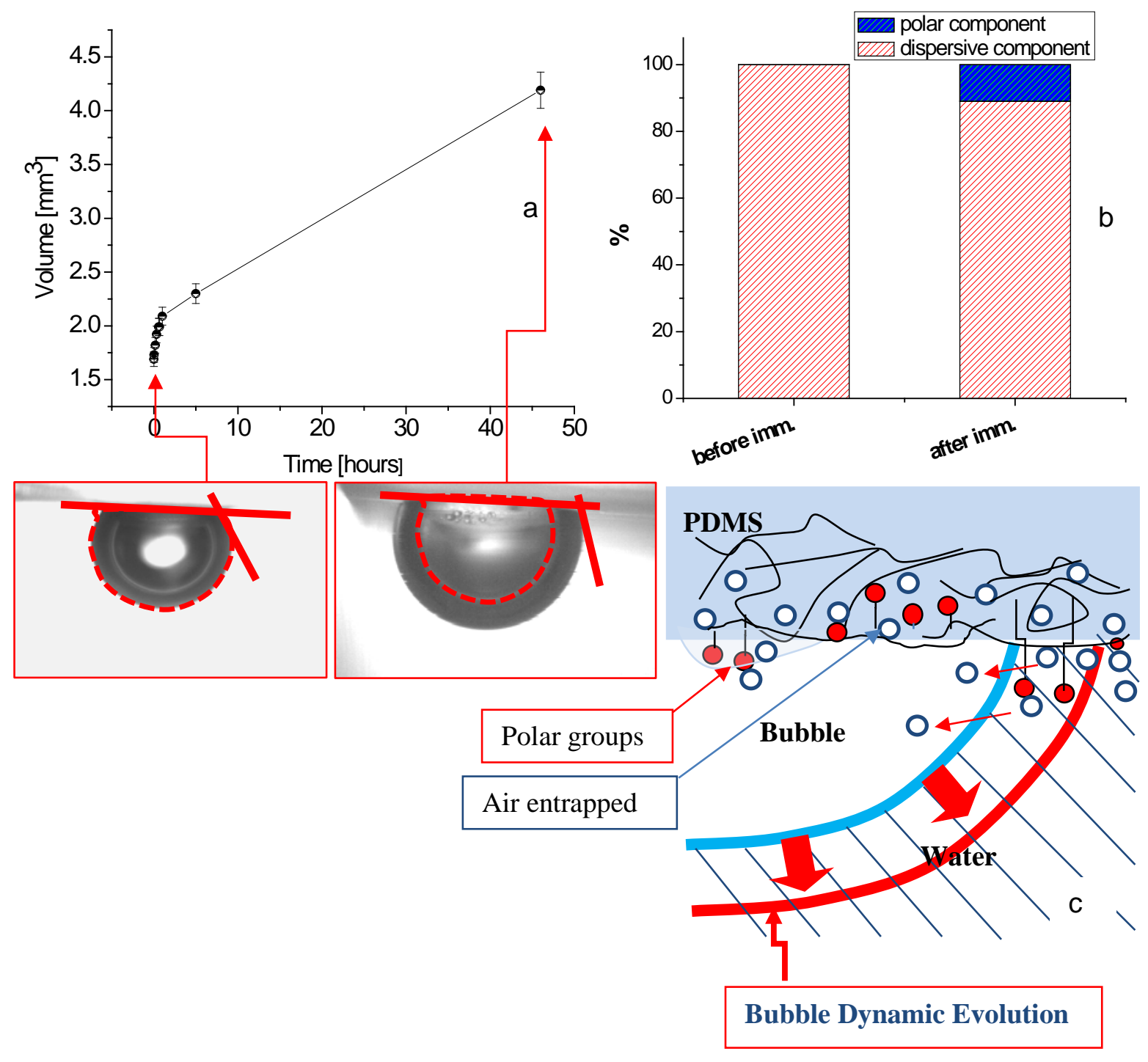

Figure 2 Variation of bubble contact angle after immersion in water (a) and variation of the polar and dispersive components before and after soaking procedure (b) on PDMS and sketch of bubble dynamic evolution on PDMS (c).

To better understand the surface modification during the immersion, we estimated the polar components present on the surface of PDMS before and after the immersion/soaking procedure using Owens - Wendt method. The results show that before immersion, the surface of PDMS was totally dispersive and after 5 hours of immersion $10 \%$ of the surface shows a polar behaviour, indicating, as reported by Kennan ${ }^{49}$ Hillbolrg et al. ${ }^{46} 27$, a migration of polar groups on the surface when the PDMS is contact with water (figure $2 \mathrm{~b}$ ). The migration of polar groups in polymer chain 
can promote the rotation and the movement of the chains of PDMS. PDMS has a porous structure, and the air is entrapped in these pores.Therefore the rearrangement and movement of polymer chain promotes the transport of the air from the polymer structure to the PDMS external surface; this process is accomplished by the percolation of water inside the first layers of PDMS due to diffusion process ${ }^{27}$. Ismail et al. ${ }^{50}$, in fact, revealed that centralized oxygen molecule from water could diffuse through methyl terminated PDMS that causes "caging" and "hopping” phenomenon. The diffusion of water in the first layers of PDMS, that is a source of degradation of PDMS backbone, can help the removing of air from polymer pores ${ }^{28}$. The air molecules that are coming from PDMS structure, due to the geometrical constraint of CCA system, (surface in contact with bubble and water under the pdms sample) are obligated to move on the surface of polymer until they nucleate in a bubble (see Figure 2.c). As a result of the rearrangement, surface structure of PDMS in water act as a small source of the flux of gas that can inflate the bubble. We noted that in the interior region of the bubble, due to the high relative humidity, there are condensed drops. (see Supporting information). The formation of a thin layer of condensed water can promote, as in immersion in water, the migration of polar groups on the polymer chains. To verify the hypothesis that the inflating mechanism is due to surface modification induced by water, we deposit a 2D layer of graphite, i.e. single-layer graphene, as a barrier on PDMS. The graphene layer was employed to a) have a rigid surface to avoid the dynamic rearrangement of the surface b) to inhibit the interaction of water and polar groups and diffusion of water. In figure 3 Raman spectra and the drop shape contact angle on PDMS coated with graphene are reported. As-deposited graphene on copper foil shows the presence of the $\mathrm{G}$ band at $\sim 1580 \mathrm{~cm}^{-1}$ (due to the first order inelastic scattering process involving the degenerate iTO and iLO phonons at the $\mathrm{G}$ point, $\mathrm{E}_{2 \mathrm{~g}}$ mode) and of the $2 \mathrm{D}$ band at $\sim 2700 \mathrm{~cm}^{-1}$ (related with the second-order zone-boundary phonons). The D peak at $\sim 1350 \mathrm{~cm}^{-1}$, related with the defectiveness of the hexagonal carbon lattice, is not revealed in the spectrum. The described features are superimposed to a luminescent behaviour, related to the metallic supporting substrate. The intensity ratio between the $G$ and 2D peaks mirrors the high quality of the graphene film. The transfer of graphene on PDMS does not dramatically affect the quality of the graphene layer. By contact angle, we observed a sensible reduction of water contact angle on graphene/PDMS, from $107^{\circ}$ to less than $100^{\circ}$, a wetting more similar to that of graphite surface. The increasing of the surface energy as indicated in Table 2, and the presence of the $G$ and 2D peaks in the Raman spectra confirm that graphene layers cover the surface. It is interesting to observe in the image photograph (inset Figure 3b), the graphene/PDMS sample preserved its optical transparency, in agreement with low optical absorption of a single layer of graphene. 


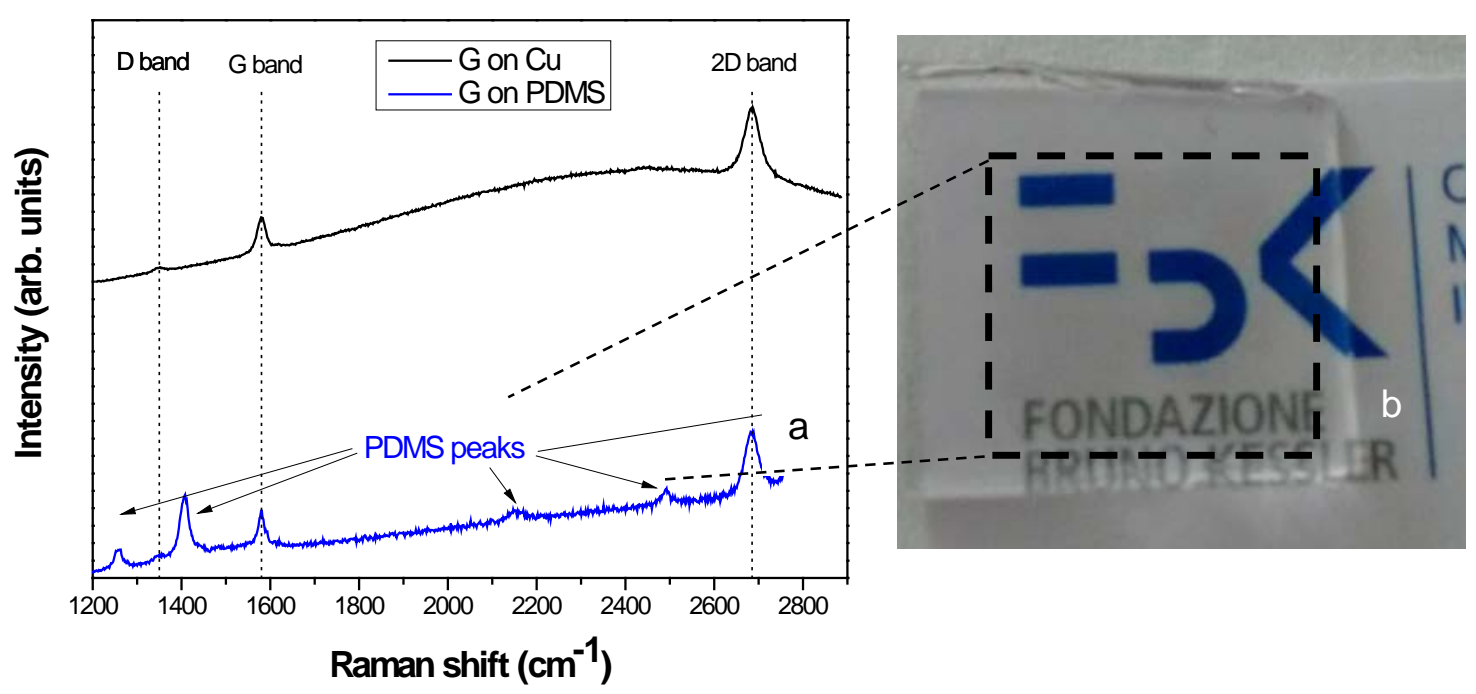

Figure 3. Raman spectra of graphene on Cu (black line) and PDMS (blue line) (a), and image of graphene/PDMS (b), graphene /PDMS sample remains transparent.

Figure 4a shows the bubble volume normalized to the initial volume of the bubble, on PDMS surface and on graphene/PDMS. The comparison clearly shows that in the system composed by graphene/PDMS the bubble size remains unchanged. The captive contact angle of graphene/ PDMS surface is reported in Figure 4b. We observed an initial increase of captive contact angle on the surface from $78^{\circ}$ to $87^{\circ}$. Similarly, to PDMS, we could expect that the variation in the early stage of the experiment is due to the contaminations ${ }^{42,43}$. After the initial stage, the CCA remains unchanged because the water cannot physically pass through the graphene barrier layer to continuously promote the migration of polar group, this is corroborated by the stabilization of bubble contact angle at $86^{\circ}$.

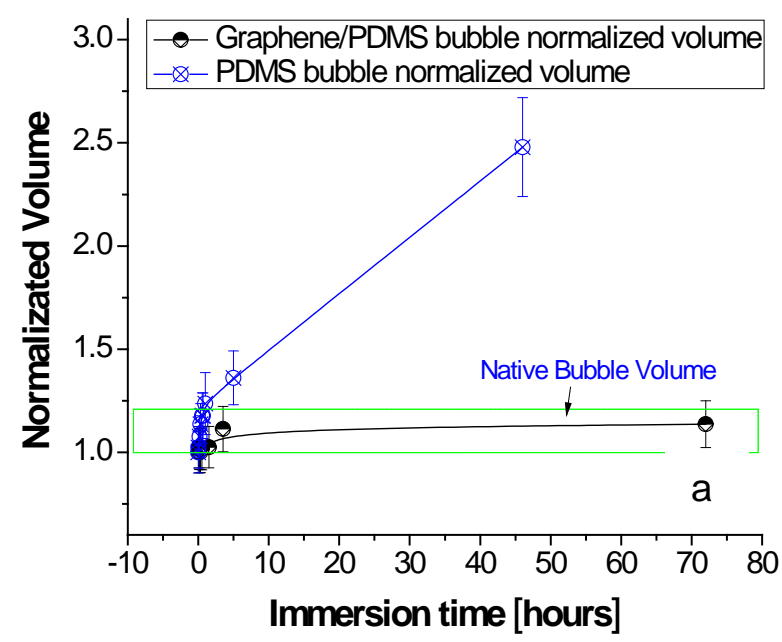


Figure 4. Bubble behavior on graphene/PDMS surface, the relative volume of the bubble as a function of immersion time(a) and CCA on graphene/PDMS (b).

To understand better the morphological changes of surfaces at the nanoscale in the water we characterized the materials using AFM equipped with a head for measurements in liquid. The interface of DI water over

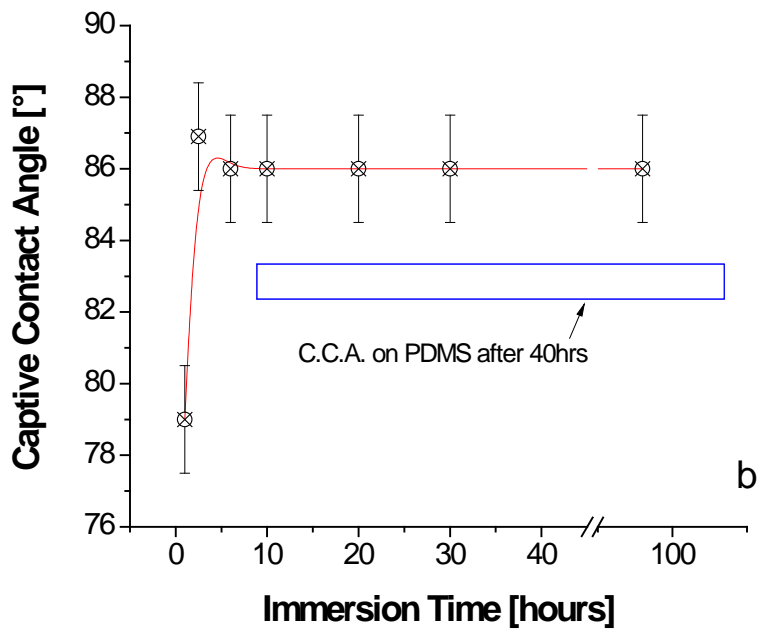
PDMS was analyzed through AFM in contact mode operation, and live imaging was carried out in water. Figure 5 is showing the AFM image of PDMS (a, b, c) and Gr/PDMS (d, e, f) in the air and water. In air condition, roughness (rms) of PDMS and Gr/PDMS was measured as $2.6 \pm 0.33 \mathrm{~nm}$ and $7.9 \pm 0.8 \mathrm{~nm}$ respectively. Higher roughness of the graphene covered PDMS is achieved by the presence of graphene wrinkles, Figure 5 (a, d). The topography of PDMS gradually changes in water medium with the progression of time. Roughness has been increased up to 5 times at different time intervals up to 48 hours after the immersion (see Supplementary information). The AFM results in combination with the result of surface polarity (figure 2.b) confirm that on PDMS there is a rearrangement of polymer chain due to the migration of polar group that induce, with inevitable diffusion of water, a sensible modification of morphology. Nevertheless, the topology of the graphene covered PDMS remain un-effected in the similar conditions. Figure 5 is showing the ability of graphene to protect the PDMS substrate from water molecules. A similar phenomenon has been observed by B. Wang et al. ${ }^{51}$ to protect silica glass surface from corrosion in water through implementation of CVD graphene as a barrier. In Figure 6 we reported for PDMS and graphene/PDMS the variation of the volume of the bubble versus the change of roughness acquired by AFM in liquid. (both parameters have been normalized to 1). We can observe that there is a direct correlation between the increase of the roughness and the dynamic evolution of bubble volume, see blue dots of PDMS surface figure 6. On graphene/PDMS, no significant variation of roughness has been observed, and no sensible change of bubble volume has been recognized 
Figure 5. AFM topography of PDMS and graphene covered PDMS (Gr/PDMS) in air and water condition after 24 and 48 hrs. Inset in panel b) shows the live imaging of the sample immersed in water.

The direct relationship between roughness evolution in water and the trend of bubble volume confirms that the dynamic evolution on PDMS is related to rearrangement of surface polymer chains as well as the diffusion of water in the polymer structure. The graphene layer inhibits almost entirely the interaction of water with PDMS surface and inhibits almost entirely the inflating of air bubble increasing the PDMS surface stability, Figure 6b. This behavior is also in line with previous reported studies on the permeation properties of single layer graphene as an absolute gas barrier ${ }^{52}$. 

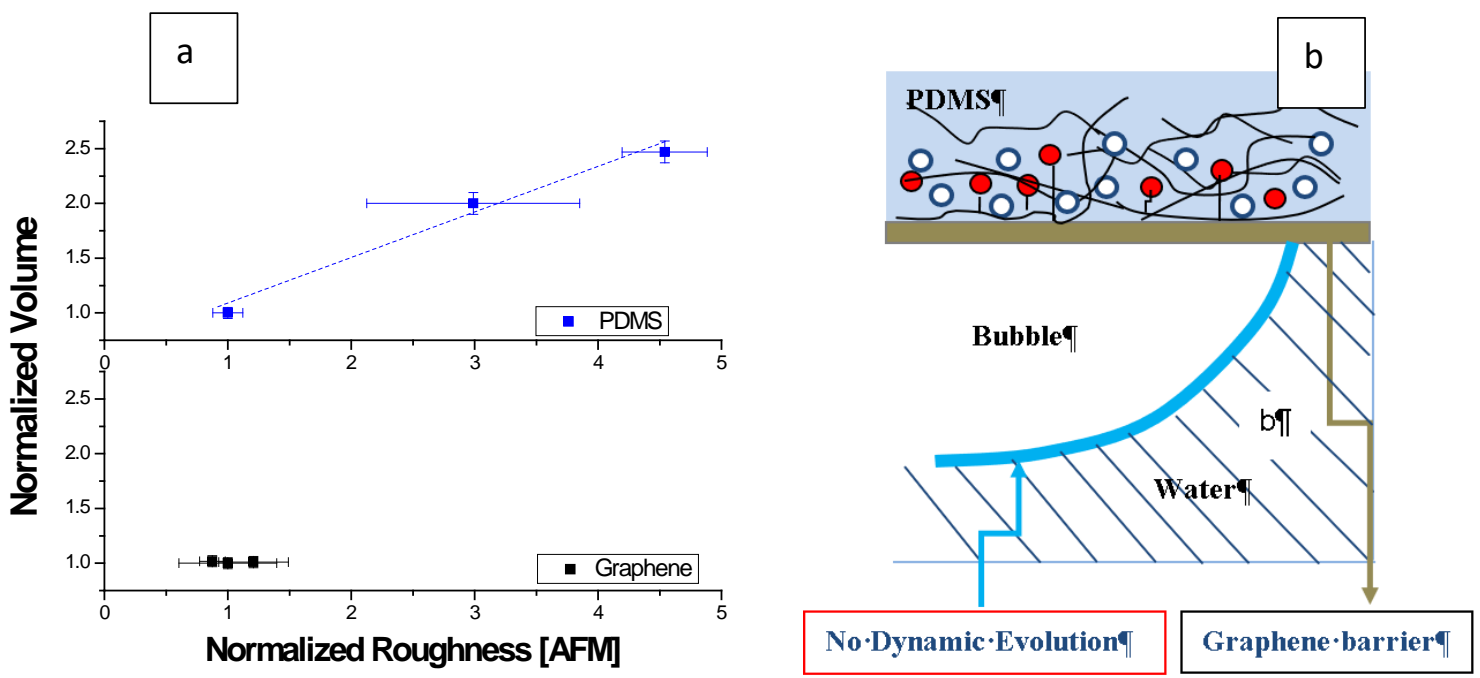

Figure 6 Variation of bubble volumes against variation of roughness during immersion in water; roughness has been estimated using AFM in liquid(a). Barrier effect of graphene (b)

We remark that even if the positive protective effect of graphene on PDMS surface was demonstrated, the full comprehension of captive contact angle and the inflating mechanism of the bubble in contact with the polymer is not reached. We did not consider, for instance, the effect of the evolution of nano/micro corrugation on the captive bubble that could play an important role in the assessment of CCA. At the nanoscale, in fact, the modification of morphology of surface can change the wetting mode, among the Wenzel mode, Penetrate mode or Cassie Baxter mode and therefore can induce different CCA. The authors underline that further investigations, in particular using computational modelling, are necessary to obtain a robust assessment of the effect of nano/micro corrugation on captive contact angle ${ }^{53}$. This is corroborated by the fact that captive contact angle is many case far by the value measured on the same material by sessile contact angle; probably the spread of liquid (penetrate model) and the spread of gas near the triple point have to be taken into consideration to obtain a more accurate description of captive contact angle. Further work is necessary to understand the dynamic behavior of the bubbles better, but we think that this topic could open new opportunities to reduce the risk of embolism in bio-implants. Moreover, we believe that the management of dynamic evolution of the bubble by means 2D materials could open a new opportunity for nano/microfluidics such as selective permeation of gas and the gas storage at the nanoscale. 


\section{Conclusion}

In this work, we studied the interaction of air bubbles with different types of dispersive surfaces. We observed that a bubble on graphite surface shows a stable behaviour. While, we observed that the bubble in contact with PDMS shows a dynamic evolution as a function of time of immersion. The results obtained on captive contact angle, the morphological evolution of polymer recognized by AFM in liquid and the variationof thermodynamical properties obtained by Owens-Wendt method indicated that this response is related to the rearrangement of the polymer structure on the surface due to the migration of polar groups on the surface. The polymer chains rearrangement on the surface of PDMS and water diffusion, that transfer the air from the bulk of polymer to the surface, behaves as supplementary source of gas that inflate the bubble in contact with the material. The experiments show that this effect can be entirely avoided using a graphene layer as a barrier without loosing the optical transparency in the visible of PDMS material. We remark that more work is necessary to understand better the progressive increase of the bubble volume. However, we believe that the effect can open new opportunity in nanofluidic fields in which air bubbles play a significant role.

\section{Supporting Information}

Further information about sessile contact angle and captive contact angle set-up, the roughness of samples immersed in water.

\section{Acknowledgement.}

N.P. is supported by the European Research Council PoC 2015 "Silkene" No. 693670, by the European Commission H2020 under the Graphene Flagship Core 1 No. 696656 (WP14 "Polymer composites") and under the Fet Proactive "Neurofibres" No. 732344.

\section{References}


1. Berdichevsky Y, Khandurina J, Guttman A, Lo YH. UV/ozone Modification of Poly(dimethylsiloxane) Microfluidic Channels. Sensors Actuators, B Chem. 2004; 97, 402408.

2. Zhang M, Feng S, Wang L, Zheng Y. Lotus Effect in Wetting and Self-Cleaning. Biotribology. 2016, 5, 31-43.

3. Gezer PG, Brodsky S, Hsiao A, Liu GL, Kokini JL. Modification of the Hydrophilic/Hydrophobic Characteristic of Zein film Surfaces by Contact with Oxygen Plasma Treated PDMS and Oleic Acid Content. Colloids Surfaces B Biointerfaces. 2016; 135, 433-440.

4. Onda T, Shibuichi S, Satoh N, Tsujii K. Super-Water-Repellent Fractal Surfaces. Langmuir. 1996;12, 2125-2127.

5. Zhao Q, Wang C, Liu Y, Wang S. Bacterial Adhesion on the Metal-Polymer Composite Coatings. Int J Adhes Adhes. 2007; 27, 85-91.

6. Bodas D, Khan-Malek C. Formation of More Stable Hydrophilic Surfaces of PDMS by Plasma and Chemical Treatments. Microelectron Eng. 2006, 83, 1277-1279.

7. Vassallo E, Cremona A, Ghezzi F, Ricci D. Characterization by Optical Emission Spectroscopy of an Oxygen Plasma Used for Improving PET Wettability. Vacuum. 2010, 84, 902-906.

8. Francioso L, De Pascali C, Bartali R, Morganti E, Lorenzelli L, Siciliano P, Laidani N,. PDMS/kapton Interface Plasma Treatment Effects on the Polymeric Package for a Wearable Thermoelectric Generator. ACS Appl Mater Interfaces, 2013, 5, 6586-6590.

9. Clair, S., Variola, F., Kondratenko, M., Jedrzejowski, P., Nanci, A., Rosei, F., \& Perepichka, D. F. Self-Assembled Monolayer of Alkanephosphoric Acid on Nanotextured Ti The Journal of Chemical Physics 2008, 128, 144705.

10. Petersson L, Meier P, Kornmann X, Hillborg H. Effect of Surface Cleanliness of Aluminium Substrates on Silicone Rubber Adhesion. J Phys D Appl Phys. 2011; 44, 034011.

11. Juárez-moreno JA, Ávila-ortega A, Oliva AI, Avilés F, Cauich-rodríguez J V. Applied Surface Science Effect of Wettability and Surface Roughness on the Adhesion Properties of 
Collagen on PDMS Films Treated by Capacitively Coupled Oxygen Plasma. Appl Surf Sci. 2015; 349, 763-773.

12. Rafiee J, Rafiee M a, Yu Z-Z, Koratkar N. Superhydrophobic to Superhydrophilic Wetting Control in Graphene films. Adv Mater. 2010; 22, 2151-2154.

13. Rafiee J, Mi X, Gullapalli H, Thomas A.V., Yabari F., ShiY., Ajayan P.M., Koratkar N.A. Wetting Transparency of Graphene. Nat Mater. 2012, 11, 217-222.

14. Drelich J, Miller JD, GooD R. The Effect of Drop ( Bubble ) Size on Advancing and Receding Contact Angles for Heterogeneous and Rough Solid Surfaces as Observed with Sessile-Drop and Captive-Bubble Techniques. J Colloid Interface Sci. 1996, 50, 37-50.

15. Maiolo D, Federici S, Ravelli L, Depero LE, Hamad-Schifferli K, Bergese P. Nanomechanics of Surface DNA Switches Probed by Captive Contact Angle. J Colloid Interface Sci. 2013, 402, 334-339.

16. Suzuki A, Eckmann DM. Embolism Bubble Adhesion Force in Excised Perfused. Anesthesiology. 2003, 9, :400-408.

17. Liao Q, Zhu X, Zheng X, Ding Y. Visualization Study on the Dynamics of CO2 bubbles in Anode Channels and Performance of a DMFC. J Power Sources. 2007, 171, 644-651.

18. Lu Y-H, Yang C-W, Fang C-K, Ko H-C, Hwang I-S. Interface-Induced Ordering of Gas Molecules Confined in a Small Space. Sci Rep. 2014; 4, 7189.

19. Sun Y, Xie G, Peng Y, Xia W, Sha J. Stability Theories of Nanobubbles at Solid-Liquid Interface: A review. Colloids Surfaces A Physicochem Eng Asp. 2016, 495, 176-186.

20. Hohm U. Experimental Static Dipole-Dipole Polarizabilities of Molecules. J Mol Struct. 2013, 1054-1055, 282-292.

21. Sun M, Luo C, Xu L, Ji H., Ouyang Q., Yu D., Chen Y. Artificial Lotus Leaf by Nanocasting. Langmuir. 2005, 21, 8978-8981.

22. Stanton MM, Ducker RE, MacDonald JC, Lambert CR, McGimpsey WG. SuperHydrophobic, Highly Adhesive, Polydimethylsiloxane (PDMS) surfaces. J Colloid Interface Sci. 2012, 367, 502-508. 
23. Preston DJ, Mafra DL, Miljkovic N, Kong J, Wang EN. Scalable Graphene Coatings for Enhanced Condensation Heat Transfer. Nanoletters. 2015, 15, 2902-2909.

24. Kozbial A, Trouba C, Liu H, Li L. Characterization of the Intrinsic Water Wettability of Graphite Using Contact Angle Measurements : Effect of Defects on Static and Dynamic Contact Angles. Langmuir. 2017, 33, 959-967.

25. Lamberti a., Quaglio M, Sacco a., Cocuzza M, Pirri CF. Surface Energy Tailoring of Glass by Contact Printed PDMS. Appl Surf Sci. 2012, 258, 9427-9431.

26. Lamberti a., Marasso SL, Cocuzza M. PDMS Membranes with Tunable Gas Permeability For Microfluidic Applications. RSC Adv. 2014, 4, 61415-61419.

27. Hillborg H, Gedde UW. Hydrophobicity Changes in Silicon Rubbers. IEEE Trans Dielectr Electr Insul. 1999; 6, 703.

28. Gustavsson TG, Gubanski SM. Hydratization of the PDMS Backbone During Water Immersion Test. IEEE-CEIDP. 1998, 269-272.

29. Bhattacharya S, Datta A, Berg JM, Gangopadhyay S. Studies on Surface Wettability of Poly(dimethyl)siloxane (PDMS) and Glass Under Oxygen-Plasma Treatment and Correlation with Bond Strength. J Microelectromechanical Syst. 2005, 14, 590-597.

30. Bodas D, Khan-Malek C. Hydrophilization and Hydrophobic Recovery of PDMS by Oxygen Plasma and Chemical Treatment-An SEM Investigation. Sensors Actuators, B Chem. 2007, 123, 368-373.

31. Hillborg H, Tomczak N, Ola A, Scho H, Vancso GJ. Nanoscale Hydrophobic Recovery: A Chemical Force Microscopy Study of UV / Ozone-Treated Cross-Linked Poly (dimethylsiloxane ) Langmuir 2004, 20, 785-794.

32. Geim AK, Novoselov KS. The Rise of Graphene. Nat Mater. 2007, 6, 183-191.

33. Stankovich S, Dikin DA, Piner RD, Kohlhaas K.A., Kleinhammes A., Jia Y., Wu Y., Nguyen S.T., Ruoff R. Synthesis of Graphene-Based Nanosheets Via Chemical Reduction of Exfoliated Graphite Oxide. Carbon N Y. 2007;45:1558-1565.

34. Ebrahimi, M., \& Rosei, F. Organic Analogues of Graphene. Nature 2017, 542, 423. 
35 Petucci, J., LeBlond, C., Karimi, M., \& Vidali, G. Diffusion, Adsorption, and Desorption of Molecular Hydrogen on Graphene and in Graphite. The Journal of Chemical Physics. 2013, 139(4), 44706.

36. Mayavan S, Siva T, Sathiyanarayanan S. Graphene Ink as a Corrosion Inhibiting Blanket for Iron in Aggressive Chloride Environment. RSC Adv. 2013, 3, 24868-24871.

37. Su Y, Kravets VG, Wong SL, Waters J, Geim a K, Nair RR. Impermeable Barrier Films and Protective Coatings Based on Reduced Graphene Oxide. Nat Commun. 2014, 5, 4843.

38. Schriver M, Regan W, Gannett WJ, Zaniewski AM, Crommie MF, Zettl A. Graphene as a Long-Term Metal Oxidation Barrier: Worse Than Nothing. ACS Nano. 2013, 7, 5763-5768.

39. Wang B, Cunning B V., Park S-Y, Huang M, Kim J-Y, Ruoff RS. Graphene Coatings as Barrier Layers to. ACS Nano. 2016, 10, 9797-9800.

40. Ghanbari-Siahkali A, Mitra S, Kingshott P. Investigation of the hydrothermal stability of cross-linked liquid silicone rubber ( LSR ), Polymer degradation and stability. 2005, 90, 471480.

41. Kozbial A, Li Z, Sun J, Gong X, Zhou F, Wang Y, Xu H, Liu H. Understanding the Intrinsic Water Wettability of Graphite. Carbon N Y. 2014, 74, 218-225.

42. Bartali R, Otyepka M, Pykal M, Lazar P, Micheli V, Gottardi G. Interaction of the Helium, Hydrogen, Air, Argon, and Nitrogen Bubbles with Graphite Surface in Water. Appl Mater Interfaces. 2017, 9, 17517-17525.

43. Li Z, Wang Y, Kozbial A, Shenoy G., Zhou F.,McGinley R, Ireland P, Morganstein B, Kunkel A, Surwade S.P, Li l, Liu H. Effect of Airborne Contaminants on the Wettability of Supported Graphene and Graphite. Nat Mater. 2013, 12, 925-931.

44. Ashraf A, Wu Y, Wang MC, Aluru NR, Dastgheib S a, Nam S. Spectroscopic Investigation of the Wettability of Multilayer Graphene Using Highly Ordered Pyrolytic Graphite as a Model Material. Langmuir. 2014; 30, 12827-12836.

45. Wei Y, Jia CQ. Intrinsic Wettability of Graphitic Carbon. Carbon N Y. 2015, 87, 10-17. 
46 Hillborg H, Ankner JF, Gedde UW, Smith GD, Yasuda HK, Wikstro K. Crosslinked Polydimethylsiloxane Exposed to Oxygen Plasma Studied by Neutron Reflectometry and Other Surface Specific Techniques. Polymer. 2000, 41, 6851-6863.

47. Thomas D.K. Scission Processes in Peroxide Cured Methylvnyl Silicone Rubber. Polymer . 1966, 7, 99-105.

48. Nagayama G. Molecular Dynamics Simulation on Bubble Formation in a Nanochannel Molecular Dynamics Simulation on Bubble Formation in a Nanochannel. Int J Heat Mass Transf. 2006; 49, 4437-4443.

49. Kennan JJ, Peters YA, Swarthout DE, Owen MJ, Namkanisorn A, Chaudhury MK. Effect of Saline Exposure on the Surface and Bulk Properties of Medical Grade Silicone Elastomers. 1996. Journal of Biomedical Materials Research. 1996, 36, 487-97.

50. Ismail AE, Grest GS, Heine DR, Stevens MJ. Interfacial Structure and Dynamics of Siloxane Systems Macromolecules 2009, 42, 3186-3194.

51. Wang B, Cunning B V., Par S-Y, Huang M, Kim J-Y, Ruoff RS. Graphene Coatings as Barrier Layers to Prevent the Water-Induced Corrosin of Silicate Glass. ACS Nano. 2016, 10, 9794-9800.

52. Koenig SP, Wang L, Pellegrino J, Bunch JS. Selective Molecular Sieving Through Porous Graphene. Nat Nanotechnol. 2012, 7, 728-732.

53. Kim D, Pugno NM, Ryu S. Wetting Theory For Small Droplets on Textured Solid Surfaces. Sci Rep. 2016, 6, 37813.

54. Rudawska A, Jacniacka E. Analysis for Determining Surface Free Energy Uncertainty By the Owen-Wendt Method. Int J Adhes Adhes. 2009, 29, 451-457.

55. Gindl M, Sinn G, Gindl W, Reiterer A, Tschegg S. A Comparison of Different Methods to Calculate the Surface Free energy of Wood Using Contact Angle Measurements. Colloids Surfaces A Physicochem Eng Asp. 2001; 181, 279-287.

56 Stalder, A. F.; Kulik, G.; Sage, D.; Barbieri, L.; Hoffmann, P., A Snake-based Approach to Accurate Determination of both Contact Points and Contact Angles. Colloids Surf., A 2006, 286, 92-103. 